\title{
Walking assessment after lumbar puncture in normal-pressure hydrocephalus: a delayed improvement over 3 days
}

\author{
Roman Schniepp, MD, ${ }^{1,2}$ Raimund Trabold, MD, ${ }^{3}$ Alexander Romagna, MD, ${ }^{3}$ \\ Farhoud Akrami, ${ }^{2}$ Kristin Hesselbarth, ${ }^{2}$ Max Wuehr, PhD, ${ }^{2}$ Aurelia Peraud, MD, ${ }^{3}$ \\ Thomas Brandt, MD, ${ }^{2,4}$ Marianne Dieterich, MD, ${ }^{1,2}$ and Klaus Jahn, MD ${ }^{2,5}$ \\ ${ }^{1}$ Department of Neurology, ${ }^{2}$ German Center for Vertigo and Balance Disorders, ${ }^{3}$ Department of Neurosurgery, ${ }^{4}$ Institute for \\ Clinical Neuroscience, Ludwig-Maximilians University Munich; and ${ }^{5}$ Schoen Klinik, Bad Aibling, Bavaria, Germany
}

\begin{abstract}
OBJECTIVE The determination of gait improvement after lumbar puncture (LP) in idiopathic normal-pressure hydrocephalus (NPH) is crucial, but the best time for such an assessment is unclear. The authors determined the time course of improvement in walking after LP for single-task and dual-task walking in iNPH.

METHODS In patients with iNPH, sequential recordings of gait velocity were obtained prior to LP (time point [TP]0), 1-8 hours after LP (TP1), 24 hours after LP (TP2), 48 hours after LP (TP3), and 72 hours after LP (TP4). Gait analysis was performed using a pressure-sensitive carpet (GAITRite) under 4 conditions: walking at preferred velocity (STPS), walking at maximal velocity (STMS), walking while performing serial 7 subtractions (dual-task walking with serial 7 [DTS7]), and walking while performing verbal fluency tasks (dual-task walking with verbal fluency [DTVF]).
\end{abstract}

RESULTS Twenty-four patients with a mean age of $76.1 \pm 7.8$ years were included in this study. Objective responder status moderately coincided with the self-estimation of the patients with subjective high false-positive results (83\%). The extent of improvement was greater for single-task walking than for dual-task walking $(p<0.05)$. Significant increases in walking speed were found at TP2 for STPS $(p=0.042)$ and DTVF $(p=0.046)$ and at TP3 for STPS $(p=0.035)$, DTS7 $(p=0.042)$, and DTVF $(p=0.044)$. Enlargement of the ventricles (Evans Index) positively correlated with early improvement. Gait improvement at TP3 correlated with the shunt response in 18 patients.

CONCLUSIONS Quantitative gait assessment in INPH is important due to the poor self-evaluation of the patients. The maximal increase in gait velocity can be observed $24-48$ hours after the LP. This time point is also best to predict the response to shunting. For dual-task paradigms, maximal improvement appears to occur later (48 to 72 hours). Assessment of gait should be performed at Day 2 or 3 after LP.

http://thejns.org/doi/abs/10.3171/2015.12.JNS151663

KEY WORDS normal-pressure hydrocephalus; gait speed; improvement; lumbar puncture; functional neurosurgery

I DIOPATHIC normal-pressure hydrocephalus (iNPH) was first described in $1965 .{ }^{1}$ The syndrome is characterized by the clinical triad of gait disturbance, cognitive dysfunction, and urinary symptoms. The prevalence of iNPH reported in selected populations and in community-based studies on adults $18-79$ years of age varies between $0.1 \%$ and $2.9 \% .{ }^{16}$ It is one of the few neurological diseases in which a gait disorder and/or dementia can improve to a complete remission of symptoms. A disturbed gait is usually the first sign and most disabling symptom. ${ }^{14}$ Typical features are a diminished gait velocity, a reduced stride length due to the co-contractions of proximal muscles, ${ }^{40}$ a reduced foot-to-floor clearance (step height) during the swing phase of the gait cycle, and increased balance-related gait measurements such as step width and foot rotation angles. ${ }^{37,39}$ Such difficulties can improve after the removal of 30-50 ml of cerebrospinal fluid (CSF) via lumbar puncture (LP). Not only is this improvement important for establishing the diagnosis, but it is also one of the few prognostic indicators of suitability for shunt implantation. ${ }^{23}$ In

ABBREVIATIONS CSF = cerebrospinal fluid; DTS7 = dual-task walking with serial 7 subtractions; DTVF = dual-task walking with verbal fluency; EI = Evans Index; iNPH = idiopathic normal-pressure hydrocephalus; LP = lumbar puncture; MMSE = Mini-Mental State Examination; MoCA = Montréal Cognitive Assessment; rm = repeated measurement; STMS = maximal walking speed; STPS = preferred walking speed; TP = time point; VR = variation rate.

SUBMITTED July 16, 2015. ACCEPTED December 10, 2015.

INCLUDE WHEN CITING Published online March 18, 2016; DOI: 10.3171/2015.12.JNS151663. 
patients who strongly respond to LP, their gait pattern often dramatically improves after shunt placement. ${ }^{15}$

Given the significance of LP in establishing the diagnosis of iNPH, the quantification of gait changes has become clinically important within the last decade. However, many studies on gait changes after LP have used clinical gait scores rather than quantitative gait analysis procedures, ${ }^{8,33}$ resulting in a limited interpretation of their findings. One quantitative study of 10 NPH patients who had undergone LP showed that gait velocity and stride length were the most responsive gait parameters, whereas cadence and balance-related parameters remained unaffected..$^{38}$ Authors of that study suggested that an increase in gait velocity by approximately $20 \%$ after LP could be considered a relevant improvement.

There is also indirect evidence that the type of quantitative gait examination strongly influences the outcome of the measurement. The importance of measuring dual motor-cognition tasks in patients with mild cognitive impairment or dementia to assess mobility problems is well-established. 3,6 This method was recently studied in an NPH cohort. ${ }^{4}$ The authors showed that patients with iNPH have reduced abilities to perform a motor-cognitive dual task. Moreover, assessing dual-task behavior was equivalent or superior to estimating the LP effect in patients with iNPH.

One pivotal requirement in assessing gait changes after LP is selecting the right time point to make such an assessment. Clearly, gait improvement after LP is a transient phenomenon, and the temporal characteristics for singleand dual-task walking can be different. Evaluating the gait performance of iNPH patients after LP at a time point of maximal improvement is desirable since it would allow an assessment with the highest sensitivity and best predictive value for shunt implantation.

However, there are few data about the time course of gait changes after LP in iNPH. Studies using nonquantitative, examiner-based gait evaluation and quantitative measures have provided evidence of early improvement within the first 24 hours after LP. ${ }^{2,33,44}$ Other studies have supported the view that gait improvement can be detected more than 24 hours after LP. ${ }^{4,38}$ Clinical standards of care usually include a follow-up assessment immediately after or the same day as the LP, although there is no consensus about the real time course of walking improvement after LP.

Until now, no study has investigated the temporal characteristics of gait improvement by using a sequential protocol of gait assessments over several days after LP. Information about the gradual improvement of gait in the days after LP would allow us to adjust the post-LP measurement to a time of optimal sensitivity for estimating the LP effect and to avoid false-negative results. Thus, we investigated gait improvement after LP over a time span ranging from the day of LP (1-8 hours after LP, Day 1) to Days 2, 3 , and 4 after LP. The temporal characteristics of gait improvement after LP were then correlated with the response to shunt surgery and to repeated LP therapy. We further compared the time course of walking improvement while performing single-task walking and while performing dual-task walking in iNPH patients after LP.

\section{Methods \\ Participants}

Patients in the departments of neurology and neurosurgery at the University Hospital of the Ludwig-Maximilians University Munich were recruited for this prospective study if they fulfilled the following clinical and radiological criteria for iNPH, as proposed by the German Neurological Society guidelines and adapted from Relkin and colleagues: ${ }^{34}$ 1) at least 2 clinical signs of the Hakim triad including gait disorder and cognitive dysfunction, 2) enlargement of the lateral ventricles (assessed using the Evans Index [EI] $>0.3$ ) on MRI or CT, 3) an opening CSF pressure $<15 \mathrm{~cm} \mathrm{H}_{2} \mathrm{O}$, and 4) exclusion of other differential diagnoses. All participants gave their written informed consent to take part in the study. The study was performed according to the guidelines laid down in the Declaration of Helsinki and its later amendments. The local ethics committee approved the study protocol.

All patients enrolled in the study underwent physical and neurological examination including testing for motor, sensory, and basal ganglia disorders. If necessary, additional complementary technical diagnostic procedures were performed, for example, electrophysiological examinations, neuroimaging of the brain or spinal cord, and vestibular testing. Cognition was assessed using either the Mini-Mental State Examination (MMSE) or the Montréal Cognitive Assessment (MoCA). ${ }^{30,43}$

\section{Lumbar Puncture Procedures}

If a suspected diagnosis of iNPH could not be rejected, patients underwent an LP procedure, during which 30-50 $\mathrm{ml}$ of CSF was removed. Gait performance was evaluated before the LP (time point [TP]0), 1-8 hours after the LP (TP1), 24 hours after LP (TP2), 48 hours after LP (TP3), and 72 hours after LP (TP4).

\section{Gait Assessment}

Gait performance was measured using a pressure-sensitive carpet system (GAITRite, CIR Systems Inc.) $6.7 \mathrm{~m}$ in length with a sampling rate of $120 \mathrm{~Hz}$. Relevant temporal and spatial gait cycle parameters were recorded, but only walking speed was analyzed. The gait analysis procedure involved a protocol of 4 different conditions: 2 speed conditions (single-task preferred walking speed [STPS] and maximal walking speed [STMS]), as well as 2 cognitive dual-task conditions (using working memory while counting backwards, that is, dual-task walking with serial 7 subtractions [DTS7]; and using semantic memory while enumerating animal names, that is, dual-task walking with verbal fluency [DTVF]). Each condition was tested twice. Each walk was started $1.5 \mathrm{~m}$ in front of the mat and continued for $1.5 \mathrm{~m}$ beyond it to provide steady-state locomotion.

After each recording, patients were asked to evaluate their gait performance ("Do you think that your gait performance has improved after LP?") by answering "yes" or "no."

\section{Data Analysis}

Walking velocity was calculated as the mean of the 2 
recordings for each condition. Matlab (The Mathworks Inc.) and SPSS (SPSS Institute Inc.) were used for data analysis. To quantify the relative change in walking speed after LP, a variation rate (VR) was calculated using the following formula:

\section{$\frac{\text { parameter after } L P \text {-parameter before } L P}{\text { parameter before } L P} \times 100$.}

The effects of the dependent variable were analyzed using a 2-way repeated measurement ( $\mathrm{rm})$ ANOVA and a Bonferroni post hoc analysis, with time points (TP1, TP2, TP3, TP4) and gait conditions (STPS, STMS, DTS7, DTVF) as factors. Significant interaction effects were further broken down into simple main effects, and the covariates of age, sex, height, and leg length were included in the model. Correlations were calculated using Pearson's and KendallTau procedures. The results were considered significant at $p<0.05$. Contingency between the self-evaluation of gait improvement and improvement according to objective gait measures was analyzed using chi-square tests.

\section{Results}

\section{Demographic Information and Clinical Features}

Twenty-four patients, 7 female and 17 male, were included in the study. Their mean age was 76.1 \pm 7.8 years, and the mean duration of the gait disorder was $35 \pm 26$ months (Table 1). All patients showed gait impairments typical of iNPH and had cognitive dysfunction (score below 25 points on the MMSE or below 26 points on the MoCA). Twenty patients had bladder dysfunction. Computed tomography or MRI revealed a mean EI of $0.39 \pm$ 0.05 , and each patient had an EI $>0.3$. The mean time period between LP and gait analysis was $3.2 \pm 2.7$ hours for TP1, $26.3 \pm 4.8$ hours for TP2, $53.7 \pm 6.4$ hours for TP3, and $74.3 \pm 9.9$ hours for TP4.

Measurement of the opening pressure after LP revealed that the intracranial pressure was below $15 \mathrm{~cm} \mathrm{H}_{2} \mathrm{O}$ for each patient. A ventriculoperitoneal shunt was placed in 10 patients at 3-12 months after the LP. All of these patients reported sustained improvement in walking performance at 3-12 months after surgery. Eight patients were treated with repeated LPs at intervals of 3-6 months. Six patients refused any further invasive treatment, for example, repeated LPs or shunt insertion. Four of those patients were treated pharmacologically with L-dopamine and memantine. Follow-up measurements of walking capacity were performed in all patients; the follow-up period ranged from 3 months to a maximum of 12 months ( 2 persons without invasive treatment).

\section{Relationship Between Subjective and Objective Measures}

The rmANOVA model did not show significant differences in the walking characteristics between the groups of "subjective responders" (patients who answered "yes" to the question, "Do you think that your gait performance has improved after LP?") and "subjective nonresponders" (patients who answered "no").

A maximum of 18 "objective responders" to LP (patients who had improvement in their gait after LP ac-
TABLE 1. Demographic and clinical characteristics of 24 patients with iNPH

\begin{tabular}{|c|c|}
\hline Variable & No. \\
\hline \multicolumn{2}{|l|}{ Basic demographic information } \\
\hline Females/males & $7: 17$ \\
\hline Mean age in yrs & $76 \pm 8$ \\
\hline Mean height in $\mathrm{m}$ & $1.74 \pm 0.12$ \\
\hline Mean duration of symptoms in yrs & $35.4 \pm 25.7$ \\
\hline \multicolumn{2}{|l|}{ Clinical feature } \\
\hline Patients w/ gait disorder & 24 \\
\hline Patients w/ bladder dysfunction & 20 \\
\hline Patients w/ cognitive dysfunction & 24 \\
\hline Mean MMSE score & $22.4 \pm 3.2$ \\
\hline Mean MoCA score & $21.5 \pm 3.1$ \\
\hline Mean ICP pressure in $\mathrm{cm} \mathrm{H}_{2} \mathrm{O}$ at LP (range) & $10.4(7-14)$ \\
\hline Mean concomitant diseases $(\mathrm{CCl})$ in points & $12.3 \pm 4.2$ \\
\hline \multicolumn{2}{|l|}{ Neuroimaging } \\
\hline Patients w/ MRI & 18 \\
\hline Patients w/ CT & 6 \\
\hline Mean El & $0.39 \pm 0.05$ \\
\hline \multicolumn{2}{|l|}{ Therapy } \\
\hline Patients w/ shunt placement & 10 \\
\hline Patients w/ repeated LP & 8 \\
\hline Patients w/ no CSF therapy & 6 \\
\hline
\end{tabular}

cording to objective measures [pressure-sensitive carpet system]) had $\geq 20 \%$ improvement in walking speed (in at least 1 condition, compared with baseline) in at least 1 post-LP measurement. Table 2 summarizes the contingency and diversity of the self-evaluation and technically based gait evaluation. Chi-square tests were not significant $(p=0.437$, chi-square $=0.605)$. Thirty-three percent $(6$ of 18) of the patients with significant objectively measured gait improvements estimated that they had a (false) negative result, whereas $83 \%$ (5 of 6 ) of the patients without significant gait improvement estimated that they had an improvement in gait (false-positive). Only 4 patients reported an immediate improvement at TP1. Fourteen patients reported an improved gait at TP2, 17 patients at TP3, and 12 patients at TP4 (Table 3).

Nine of the 17 subjective responders underwent shunt implantation; 1 patient who did not subjectively improve (but showed an increased walking speed during gait analysis) underwent surgery 10 months after LP.

TABLE 2. Contingency table for self-evaluation versus objective gait analysis

\begin{tabular}{|c|c|c|c|}
\hline \multirow{2}{*}{$\begin{array}{c}\text { Objective Gait } \\
\text { Assessment }\end{array}$} & \multicolumn{2}{|c|}{ Self-Evaluation } & \multirow[b]{2}{*}{ Results } \\
\hline & Improved & Not Improved & \\
\hline Improved ( $\geq 20 \%)$ & 12 & 6 & FN 6/18, TP 12/18 \\
\hline Not improved $(<20 \%)$ & 5 & 1 & FP 5/6, TN $1 / 6$ \\
\hline
\end{tabular}




\section{Temporal Consistency of the Gait Measures}

Individual comparisons of the objective responder status at each time point revealed a high inner-subject consistency of the data. Table 3 summarizes the absolute numbers of objective responders and the patients who switched (called "converters") from responder to nonresponder status or vice versa between the subsequent time points. Converters from nonresponder to responder status were mainly observed between TP1, TP2, and TP3, according to the idea of gradual gait improvement after LP. Converters from responder to nonresponder status were mainly observed between TP3 and TP4, indicating a weakening of the LP effect after 48 hours. No patients switched the responder status more than 1 time within the 3 days after measurement. A maximum of 2 patients converted from responder to nonresponder status between TP2 and TP3 for the condition of STMS (only 1 of those also at the other conditions).

\section{Dual-Task Performance}

Walking speeds during DTS7 $(\mathrm{F}=4.467, \mathrm{p}=0.024)$ and DTVF ( $\mathrm{F}=4.239, \mathrm{p}=0.028)$ were significantly decreased compared with those during STPS (at TP0). These differences remained at TP1 and TP2 but diminished at TP3 and TP4. The mean dual-task costs (that is, the percentage decrease in walking speed during dual-task walking) were $-28.5 \% \pm 18.9 \%$ for DTS7 and $-28.1 \% \pm 15.8 \%$ for DTVF at TP0.

There were no correlations between baseline walking performance and the individual dual-task costs, but there was a strong correlation between the individual dual-task costs for both dual tasks $\left(\mathrm{R}^{2}=0.94, \mathrm{p}<0.001\right)$.

\section{Walking Velocities After LP}

In the rmANOVA model, there was a significant increase in walking velocity after LP for the walking conditions of STPS, DTS7, and DTVF (Table 4). Bonferroni post hoc analysis of the interaction effect (walking condition $\times$ time point) revealed a significant increase in walking speed (compared with TP0) at TP2 for STPS ( $\mathrm{F}=3.96$, $\mathrm{p}=0.042)$ and for DTVF $(\mathrm{F}=3.52, \mathrm{p}=0.046)$. At TP3, there were significant increases in walking speed (compared with TP0) for STPS $(\mathrm{F}=4.11, \mathrm{p}=0.035)$, for DTS7 $(F=3.98, p=0.042)$, and for DTVF $(F=3.76, p=0.044)$.

The rmANOVA model of the VRs in walking speed revealed a significant increase after LP for all 4 walking conditions (Table 4 and Fig. 1).

\section{Evans Index and Gait Performance in iNPH}

The EIs of the iNPH patients were increased (mean of 0.39 , range $0.32-0.45$ ); thus, every patient met the criterion of ventricle enlargement (EI > 0.30). There was no significant correlation between the EIs and the absolute values of gait velocity at any time point of the gait measurements. Significant positive correlations for STPS and STMS were found for the VRs in walking speed. The EIs positively correlated with the VRs in walking speed at TP1 for STPS $\left(\mathrm{R}^{2}=0.693, \mathrm{p}=0.0001\right.$; Fig. 2$)$ and for STMS $\left(R^{2}=0.406, p=0.049\right)$. At TP2, there was a positive correlation between the VRs in walking speed and the EIs for STPS only $\left(\mathrm{R}^{2}=0.612, \mathrm{p}=0.001\right.$; Table 5$)$.

\section{Relationship Between LP Response and Therapeutic Outcome}

The improvement in walking speed after therapy significantly depended on the therapeutic option chosen. The greatest improvement in walking speed was found for shunt surgery (for example, $34.7 \% \pm 11.1 \%$ for STPS), followed by the repeated LP strategy (for example, 17.2\% $\pm 18.8 \%$ for STPS). Patients who refused further CSF removal showed a gradual decrease in walking speed (for example, $-28.1 \% \pm 13.2 \%$ for STPS). The administration of dopamine and memantine did not completely reverse this decline (for example, $-16.2 \% \pm 6.2 \%$ for STPS). Parallel results were present in the other walking conditions (Supplemental Table 1). Post hoc analysis of the rmANOVA model showed that shunt surgery and repeated LPs led to significantly greater improvements in gait velocity compared with those in patients without CSF removal $(\mathrm{p}<0.05)$. The response to LP correlated with improvement after shunt surgery and under repeated CSF removals. However, correlations between the VRs in walking speed improvement after LP and the VRs in walking speed improvement under CSF removal therapy (either shunt or repeated LPs) were only significant for delayed time points at TP3 (for STPS, for STMS, and for DTS7, all $p<0.05$; Supplemental Table 2 ). No correlation was found for the EIs and gait improvements during follow-up.

\section{Discussion}

This study provides the first evidence of a delayed change in gait speed in patients with iNPH during the first 3 days after LP. Our main findings are as follows: 1) A reduction in gait velocity is a key feature of the iNPH gait disorder, and the removal of CSF improves walking ve-

TABLE 3. Time course of subjective and objective gait improvement after LP

\begin{tabular}{ccccc}
\hline Gait Improvement & TP1 & TP2 & TP3 & TP4 \\
\hline Subjective responder & 4 & 14 & 17 & 12 \\
\hline Objective responder & Responder/converter (R/NR) & Responder/converter (R/NR) & Responder/converter (R/NR) & Responder/converter (R/NR) \\
\hline STPS & $8 /(0 / 0)$ & $16 /(8 / 0)$ & $16 /(1 / 1)$ & $7 /(1 / 10)$ \\
\hline STMS & $2 /(0 / 0)$ & $7 /(7 / 2)$ & $12 /(5 / 0)$ & $7 /(2 / 7)$ \\
\hline DTS7 & $8 /(0 / 0)$ & $11 /(4 / 1)$ & $19 /(8 / 0)$ & $13 /(1 / 7)$ \\
\hline DTVF & $9 /(0 / 0)$ & $12 /(4 / 1)$ & $18 /(7 / 1)$ & $13 /(1 / 6)$ \\
\hline
\end{tabular}

$\mathrm{NR}=$ nonresponder; $\mathrm{R}=$ responder.

* Converter status mentioned as converted to responder and converted to nonresponder. 
TABLE 4. Walking speed improvement after LP for the tested walking paradigms*

\begin{tabular}{|c|c|c|c|c|c|c|}
\hline Condition & TPO & TP1 & TP2 & TP3 & TP4 & rmANOVA \\
\hline \multicolumn{7}{|c|}{ Walking velocity (m/sec) } \\
\hline STPS & $0.59 \pm 0.09$ & $0.64 \pm 0.10$ & $0.72 \pm 0.11$ & $0.77 \pm 0.09$ & $0.64 \pm 0.11$ & $F 4,23=3.04, p<0.020$ \\
\hline STMS & $0.93 \pm 0.20$ & $0.99 \pm 0.21$ & $1.00 \pm 0.19$ & $1.09 \pm 0.21$ & $0.97 \pm 0.22$ & $\mathrm{~F} 4,23=0.82, p=\mathrm{NS}$ \\
\hline DTS7 & $0.42 \pm 0.09$ & $0.47 \pm 0.12$ & $0.52 \pm 0.11$ & $0.58 \pm 0.09$ & $0.54 \pm 0.10$ & $F 4,23=2.71, p<0.048$ \\
\hline DTVF & $0.41 \pm 0.06$ & $0.46 \pm 0.10$ & $0.53 \pm 0.13$ & $0.58 \pm 0.12$ & $0.54 \pm 0.11$ & $F 4,23=2.72, p<0.048$ \\
\hline \multicolumn{7}{|c|}{ VRs for walking velocity (\%) } \\
\hline STPS & - & $12.1 \pm 6.0$ & $26.2 \pm 17.5$ & $36.8 \pm 13.6$ & $12.5 \pm 13.3$ & $F 3,23=3.85, p<0.012$ \\
\hline STMS & - & $7.5 \pm 11.1$ & $10.5 \pm 15.6$ & $20.3 \pm 22.5$ & $6.2 \pm 19.5$ & $F 3,23=3.21, p<0.020$ \\
\hline DTS7 & - & $14.3 \pm 11.5$ & $32.3 \pm 20.5$ & $52.1 \pm 15.9$ & $43.6 \pm 24.6$ & $F 3,23=3.25, p<0.018$ \\
\hline DTVF & - & $10.9 \pm 24.4$ & $36.4 \pm 26.6$ & $50.4 \pm 21.4$ & $40.2 \pm 24.2$ & $F 3,23=2.85, p<0.042$ \\
\hline
\end{tabular}

NS $=$ not significant, $p>0.05$.

* The effect of removing $40 \mathrm{ml}$ of CSF on the walking velocity of patients with iNPH. Values expressed as the mean \pm standard deviation of the raw values for gait speed and the percentage improvement in gait speed over a time span of 90 hours after LP. Boldface type indicates a significant post hoc Bonferroni correction for the time point.

locities during single- and dual-task walking conditions. 2) Significant improvements in walking speed were identified 24-48 hours after LP (maximum). 3) Ventricular enlargement correlated with a relative improvement in walking speed in the early phase after LP. 4) The amount of walking improvement 48-72 hours after LP correlated with the benefit from CSF removal therapy (shunt or repeated LP).

\section{Gait Assessment as a Key Procedure for LP and iNPH}

Clinical and radiological signs of iNPH are fundamental for establishing a diagnosis of iNPH and selecting can-

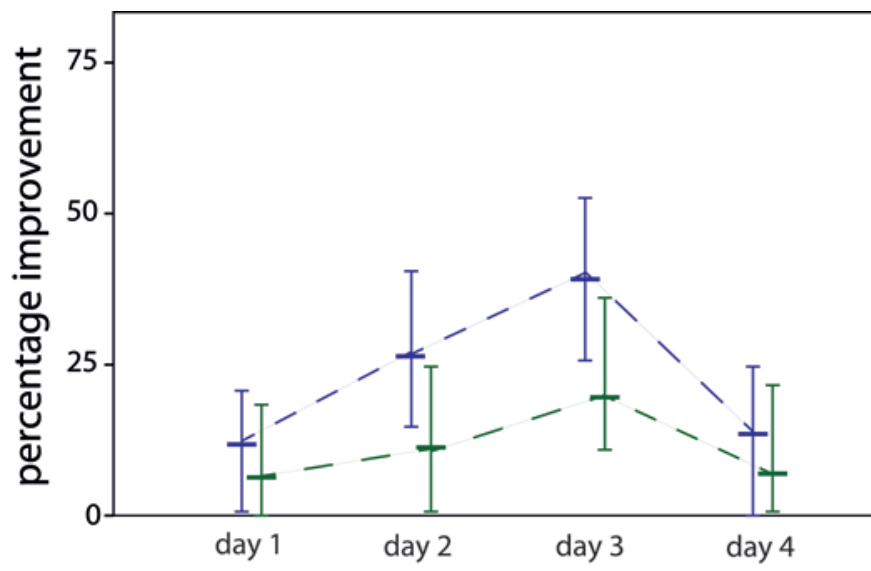

- single task preferred speed
- single task fast speed

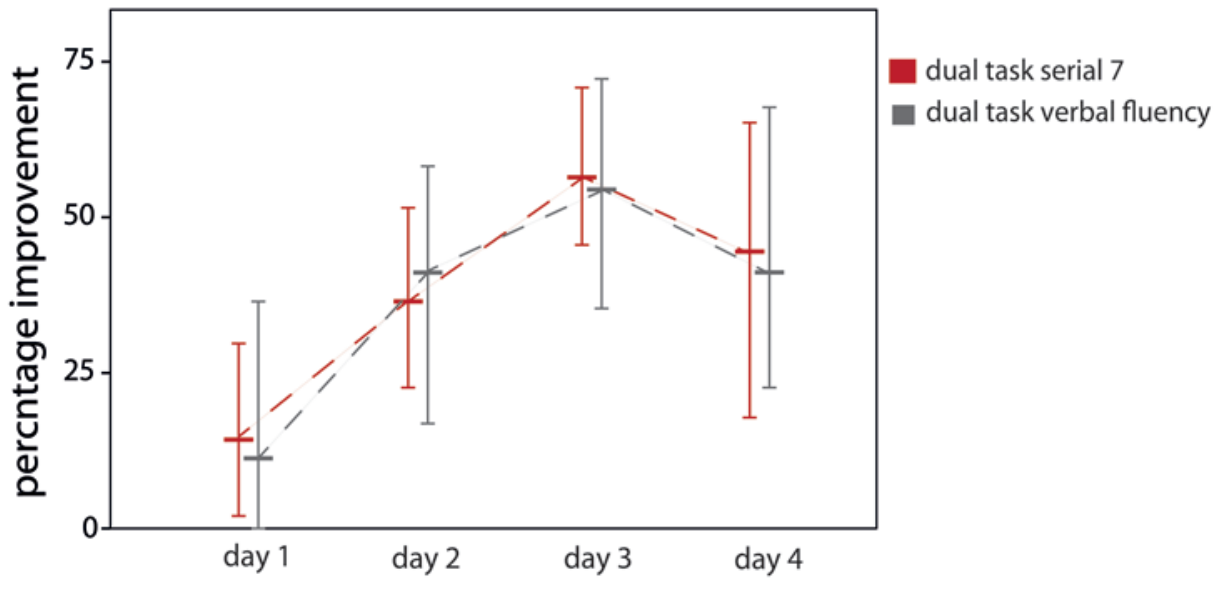

FIG. 1. Time course of relative changes in walking speed after LP. Mean values with upper and lower quartiles of the percentage improvement (VRs) in walking speed following the LP procedure, which involved the removal of $30-50 \mathrm{ml}$ of CSF. Figure is available in color online only. 


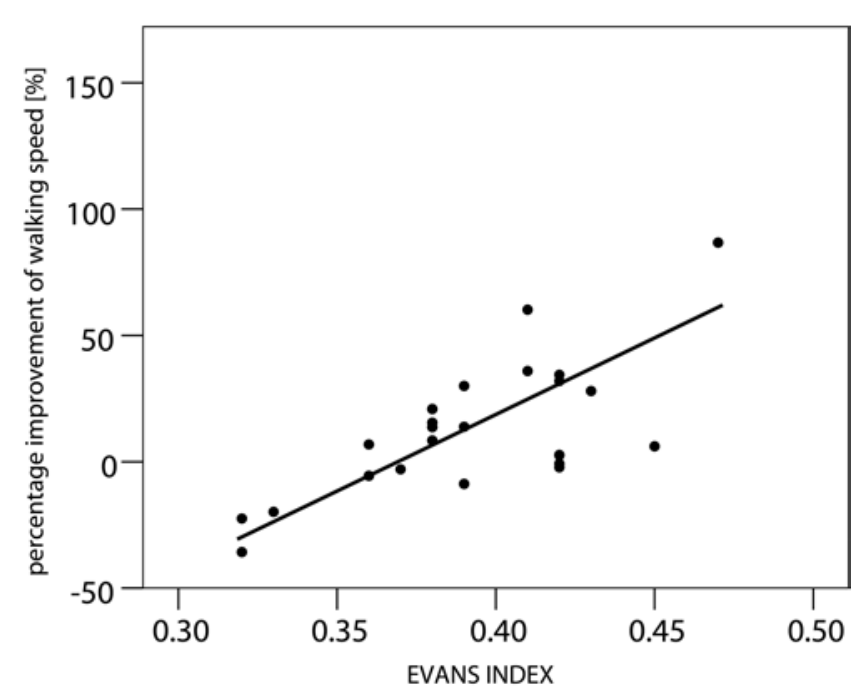

FIG. 2. Pearson's correlation of the El and the VRs in walking speed improvement on the day of $L P\left(1-8\right.$ hours after LP). $R^{2}=0.693, p<0.001$.

didates for shunt placement. A positive response to LP is the most common clinical sign used to verify the diagnosis and to predict a beneficial outcome of shunt surgery. ${ }^{47}$ Quantitative gait assessment helps by providing standardized and comparable data for the clinician..$^{38}$ The importance of such quantitative measures for evaluating LP is further stressed by the low reliability of patient self-evaluations. Only 54\% of the patients had subjective outcomes consistent with the objective gait measures. Obviously, there is a strong tendency to subjectively overestimate gait performance after LP. Increased levels of motivation and the anticipation of possible treatment options after a positive LP outcome could lead to such false-positive selfevaluations. Since a positive LP outcome serves as a strong argument for shunt insertion in iNPH, clinicians should assess gait in an objective way to avoid false-positive evaluations of the LP procedure.

While the patient's appraisal of an improvement in gait after LP is decisive in delivering treatment for iNPH, it is still subject to false results. Quantitative gait measures, however, provide objective measures of the clinical outcome that could be used to identify false-positive responders and avoid exposing this subgroup to surgical risks.

\section{Walking Speed Reduction as a Key Aspect of the iNPH Gait Disorder}

It has been demonstrated that a reduced walking pace is a key feature of the gait disorder in patients with iNPH..$^{38}$ This clinical hallmark can be easily assessed. In agreement with previous studies, the iNPH cohort in the current study showed a preferred walking pace that was approximately $50 \%$ of the walking pace of healthy subjects. ${ }^{46} \mathrm{Re}-$ duced walking speed is a key feature of mobility impairments and has been linked to a decreased quality of life, decreased mobility, and impaired everyday independence of elderly persons. ${ }^{27,28}$ Stolze et al. suggested that an improvement in walking speed after LP by $\geq 20 \%$ could be considered a relevant improvement. ${ }^{38}$ This threshold was used in the present study to define the objective responder status, and the maximum average walking speed improvement was 37\% at TP2 for STPS. During STMS, the percentage of improvement was lower. Thus, patients with iNPH increased their self-selected walking pace, but their maximal speed capacity was only slightly improved.

\section{Dual-Task Performance of Patients With iNPH}

Currently, postural control is believed to share attentional resources with cognition. ${ }^{17}$ Dual-task paradigmsthat is, the simultaneous tasks of walking and cognitionhave been used to shed light on motor and cognitive interference during gait. ${ }^{48}$ The walking and cognitive functions of patients with iNPH are known to be independently improved after LP. ${ }^{37,42}$ In a recent study, spatiotemporal gait parameters assessed during dual-task walking were proven to be a good discriminator of responders versus nonresponders in patients with iNPH after LP. ${ }^{4}$

Our results support and extend these findings, showing that 22 of 24 patients with iNPH walked more slowly during the dual task than during the single task. The average dual-task cost of approximately $28 \%$ is comparable to findings reported in a recent study ${ }^{5}$ and other clinical cohorts. ${ }^{10,19}$

The comparison of dual-task costs between the serial 7 task and the verbal fluency task shows a strong inter-task correlation; thus, the type of cognitive task is not relevant for the result of the dual-task gait assessment. Instead, impaired dual-task performance reflects a disturbance in higher-level processing domains (for example, attention-

TABLE 5. Evans Indexes and walking performance in iNPH patients*

\begin{tabular}{|c|c|c|c|c|c|}
\hline Condition & TPO & TP1 & TP2 & TP3 & TP4 \\
\hline \multicolumn{6}{|l|}{ El \& walking speed } \\
\hline STPS & NS & NS & NS & NS & NS \\
\hline STMS & NS & NS & NS & NS & NS \\
\hline DTS7 & NS & NS & NS & NS & NS \\
\hline DTVF & NS & NS & NS & NS & NS \\
\hline \multicolumn{6}{|c|}{ El \& VRs in walking speed } \\
\hline STPS & - & $0.693, p=0.000$ & $0.612, p=0.001$ & NS & NS \\
\hline STMS & - & $0.406, p=0.049$ & NS & NS & NS \\
\hline DTS7 & - & NS & NS & NS & NS \\
\hline DTVF & - & NS & NS & NS & NS \\
\hline
\end{tabular}

* Results of Pearson's correlation procedures of individual Els and gait velocities and the improvement in gait velocities (expressed in VRs). 
dividing problem $)^{32}$ in iNPH that results in an inferior gait performance.

Studies on gait impairment due to small vessel disease of the brain revealed the impact of predominantly frontal white matter alterations and stressed the fundamental role of frontal brain regions in the cognitive control of motor performance (for example, the anterior thalamic radiation $\left.^{7,13,36}\right)$. We found no relationship between the EI and the dual-task cost in our data, a result that suggests that ventricular enlargement alone cannot explain the dual-task impairments in iNPH. Metabolic, vascular, or other disturbances of deep white matter in the frontal brain regions may explain the dual-task problems of patients with iNPH; however, our study design does not allow us to speculate on any underlying pathophysiological mechanisms. Further studies using more sophisticated neuroimaging techniques are necessary to investigate brain structure and/or function relationships in iNPH.

Analysis of the improvement in walking speed after LP revealed greater relative improvements for dual-task walking than for single-task walking. However, this effect may be a result of the lower baseline values of gait speed at TP0. In contrast to the earlier findings of Allali et al., ${ }^{4}$ our absolute improvements for single-task walking were higher than those for dual-task walking. In particular, for a clinical setup, when the calculation of small absolute differences in relative changes is difficult, it may be better to assess iNPH walking performance in a single-task procedure. If single-task walking is only slightly affected in patients with iNPH, the addition of dual-task walking paradigms may help to avoid ceiling effects for the LP results.

\section{Temporal Characteristics of Gait Improvement in iNPH After LP}

The improved walking of patients with iNPH after LP is a transient phenomenon. An earlier study provided evidence of improvement within the first 24 hours after LP; ${ }^{44}$ however, subsequent time points more than 24 hours after LP were not investigated. Our study is the first systematic investigation of the time course of gait improvement within the first 72 hours after LP. The results of the singletask and dual-task walking assessments indicated that the maximum gait improvement can be detected 24-72 hours after LP. Nearly half of the objective responders according to our definition of a responder status would have been missed within the first 24 hours after LP. This finding indicates that assessment of gait speed changes within the first 24 hours after LP is susceptible to false-negative results. Apart from the gradual improvement over 48-72 hours, the inner-subject consistency for subsequent measurements was high. Only 2 patients had conflicting results over the time span measured. Thus the improvement in walking speed reflects a robust outcome measure of the LP effect.

There is evidence that the extent of gait improvement after LP corresponds to the extent of the benefit after shunt surgery. ${ }^{12,18}$ This association was present in all patients with iNPH who had undergone CSF removal therapies, either shunt surgery or repeated LPs. Significant correlations between the LP response and therapy outcomes, however, were only present for gait assessments at late time points (that is, 48-72 hours after LP). This finding further indicates that the delayed improvement in gait velocity after a single LP could have higher predictive power than the results of early assessments. Accordingly, clinicians should measure the effects on walking performance after LP at the time of maximum change to adequately estimate the capacity for improvement after therapy.

Pathophysiological models of the gait disorder in iNPH suggest a direct compression or deformation of the motor fibers in the medial portion of the corona radiata. ${ }^{35}$ The removal of CSF may inhibit the pathological high pressure gradients in the lateral and third ventricles and the sylvian fissures, ${ }^{22}$ which could lead to a decompression of frontal and/or mesencephalic fibers of motor or premotor areas. Other studies provide evidence for increases in regional blood flow in different brain areas of patients with iNPH after LP., ${ }^{911,20,31,45}$ These mechanisms would explain an immediate release of symptoms after CSF removal. In the current study, 8 patients showed slight gait improvements immediately after LP. Moreover, the observed significant correlation between the extent of lateral ventricle enlargement (measured by the EI) and early gait improvement further supports the pressure-related symptom release in iNPH. However, the current study also has implications for delayed alternative and/or complementary pathophysiological processes, which could be involved in the LP response. Momjian et al. described the relationship between reduced cerebral blood flow in the deep white matter of patients with iNPH and impaired cerebral vascular autoregulation. ${ }^{29}$ Patients with iNPH were shown to have disturbed autoregulation of cerebral arteries, especially near the lateral ventricles. Impaired autoregulation of the arteries could led to edema and local ischemia, which were thought to contribute to the emergence of the gait disorder in iNPH. Lumbar puncture-associated changes in the CSF pressure gradient at the lateral ventricle walls and in the surrounding white matter were shown to lead to a shift in the watershed phenomenon of the regional blood flow, thus improving vascular autoregulation in the periventricular areas. ${ }^{25,26}$ Moreover, changes in the interstitial fluid pressure by CSF removal could decrease edema in the periventricular tissue, ${ }^{24,41}$ thereby improving the clearance of vasoactive and/or neurotoxic metabolites. Both the improved vascular autoregulation and the enhanced capacity for metabolic clearance may be the pathophysiological substrates of delayed walking improvement in the first days after LP. ${ }^{21}$

However, the current study was not designed to adequately address the relationship between brain structure and function in iNPH. Future investigations using neuroimaging and neurophysiological measurements in parallel are required to directly address these pathophysiological mechanisms.

\section{Recommendations for a Clinical Gait Assessment During the LP Procedure}

Subjective estimations of gait improvement by patients with iNPH are prone to false-negative and false-positive ratings. Quantitative gait measurements can help to improve the sensitivity and predictive value of the LP procedure: Gait velocity (and stride length) can be easily 


\section{pre LP evaluation}

walking test $25 \mathrm{ft}$./ $8 \mathrm{~m}$.

single task (preferred and fast walking)

dual task (Serial 7/ Verbal Fluency)

\section{post LP evaluation}

walking test $25 \mathrm{ft}$./ $8 \mathrm{~m}$.

single task (preferred and fast walking)

dual task (Serial 7/ Verbal Fluency)

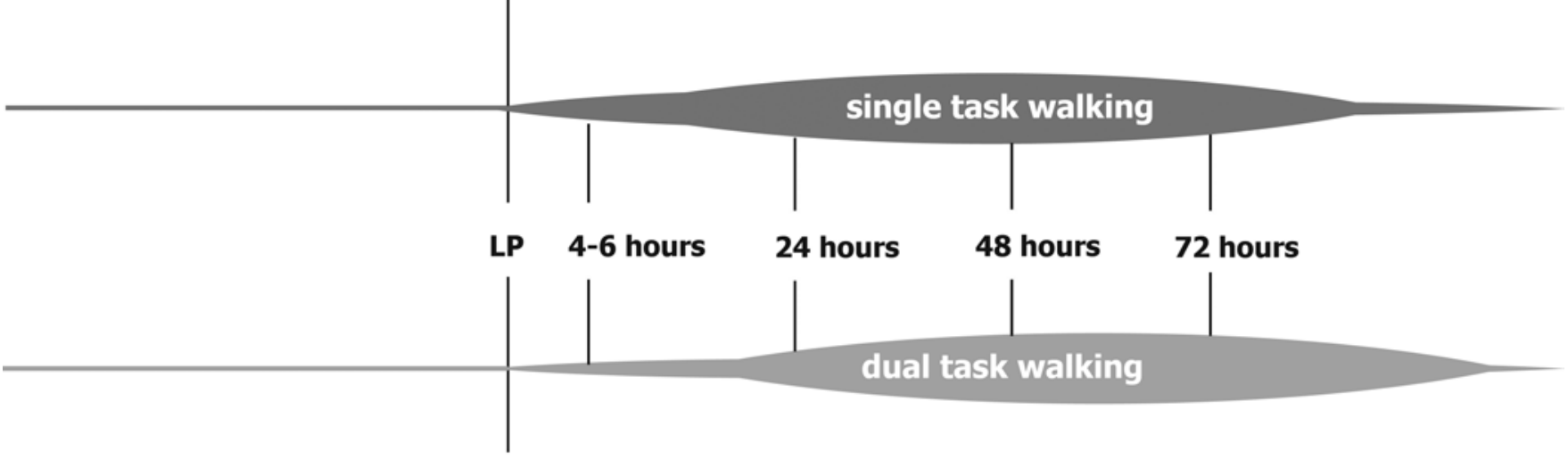

FIG. 3. Schematic of the time course of gait improvement after CSF removal (removal of 30-50 ml CSF) in patients with iNPH. Schematic of gait velocity improvement after LP. Gait velocity can be easily assessed on a $25-\mathrm{ft} / 8-\mathrm{m}$ walking test. Single-task walking assessment should include walking with preferred speed and with fast speed to obtain information about the overall walking capacity and the self-selected utilization of this capacity. The performance of dual tasks with executive (serial 7) and verbal fluency tasks (naming words) helps to identify attentional resources during walking and to simulate everyday mobility tasks. Walking performance in iNPH improves directly after the LP: there is a gradual improvement for 24-48 hours (for single task). Single-task walking improvement appears earlier than dual-task walking improvement.

assessed using a 35-ft (8-m) walking test, during which the physician records the ambulation time and the total steps. To assess walking speed capacity and the individual utilization of preferred speed, single-task walking with preferred speed and with maximally fast speed should be measured. We recommend performing a dual-task walking examination by adding an executive task (serial 7) and/or verbal fluency tasks (naming words) for patients with only minor impairments during single-task walking. Moreover, these conditions help to identify the extent of attentional resources spent on walking and simulate everyday mobility tasks.

The evaluation of post-LP measurements should be done not only 2-6 hours after the LP (to assess direct improvement) but also between 24 and 48 hours after the LP to avoid false-negative results and to estimate the real extent of walking improvement (Fig. 3).

\section{Limitations of the Study}

This study has several limitations. The study cohort of 24 patients with iNPH is relatively small for estimating correlations between the follow-up outcomes and the LP response. With the 18 patients who were treated with either shunt surgery or repeated LPs, we have reached a sufficient sample size for the Pearson's correlations. For correlations with other outcome variables, such as quality of life, mobility measures, or independency, a further systematic study is necessary.

Moreover, the time points of follow-up measurements differed from 3 to 12 months. With respect to a possible dynamic disease course, this heterogeneity in follow-up times may lead to deficient interpretation of the followup results; however, only 2 patients (both with a nonCSF removal therapy) had a late follow-up measurement.
The analysis of associations between LP results and gait outcomes after shunt insertion or repeated LPs was performed with a very homogeneous follow-up period of 3-6 months after therapy.

\section{Conclusions}

The evaluation of post-LP measurements in iNPH should be done not only 2-6 hours after the LP (to assess direct improvement) but also between 24 and 48 hours after the LP to avoid false-negative results and to estimate the real extent of walking improvement.

\section{Acknowledgments}

This work was supported by the German research foundation (Deutsche Forschungsgemeinschaft, DFG JA 1087/1-1), the German Hertie Foundation, and the German Federal Ministry for Education and Science (BMBF, Nr. 01EO0901).

We thank Judy Benson for copyediting the manuscript.

\section{References}

1. Adams RD, Fisher CM, Hakim S, Ojemann RG, Sweet WH: Symptomatic occult hydrocephalus with "normal" cerebrospinal-fluid pressure: a treatable syndrome. N Engl J Med 273:117-126, 1965

2. Agostini V, Lanotte M, Carlone M, Campagnoli M, Azzolin I, Scarafia R, et al: Instrumented gait analysis for an objective pre-/postassessment of tap test in normal pressure hydrocephalus. Arch Phys Med Rehabil 96:1235-1241, 2015

3. Allali G, Kressig RW, Assal F, Herrmann FR, Dubost V, Beauchet $\mathrm{O}$ : Changes in gait while backward counting in demented older adults with frontal lobe dysfunction. Gait Posture 26:572-576, 2007

4. Allali G, Laidet M, Beauchet O, Herrmann FR, Assal F, Armand S: Dual-task related gait changes after CSF tapping: a 
new way to identify idiopathic normal pressure hydrocephalus. J Neuroeng Rehabil 10:117, 2013

5. Armand S, Allet L, Landis T, Beauchet O, Assal F, Allali G: Interest of dual-task-related gait changes in idiopathic normal pressure hydrocephalus. Eur J Neurol 18:1081-1084, 2011

6. Beauchet O, Freiberger E, Annweiler C, Kressig RW, Herrmann FR, Allali G: Test-retest reliability of stride time variability while dual tasking in healthy and demented adults with frontotemporal degeneration. J Neuroeng Rehabil 8:37, 2011

7. Benson RR, Guttmann CR, Wei X, Warfield SK, Hall C, Schmidt JA, et al: Older people with impaired mobility have specific loci of periventricular abnormality on MRI. Neurology 58:48-55, 2002

8. Bugalho P, Guimarães J: Gait disturbance in normal pressure hydrocephalus: a clinical study. Parkinsonism Relat Disord 13:434-437, 2007

9. Capel C, Kasprowicz M, Czosnyka M, Baledent O, Smielewski P, Pickard JD, et al: Cerebrovascular time constant in patients suffering from hydrocephalus. Neurol Res 36:255-261, 2014

10. Coelho FG, Stella F, de Andrade LP, Barbieri FA, SantosGalduróz RF, Gobbi S, et al: Gait and risk of falls associated with frontal cognitive functions at different stages of Alzheimer's disease. Neuropsychol Dev Cogn B Aging Neuropsychol Cogn 19:644-656, 2012

11. Czosnyka ZH, Czosnyka M, Whitfield PC, Donovan T, Pickard JD: Cerebral autoregulation among patients with symptoms of hydrocephalus. Neurosurgery 50:526-533, 2002

12. Damasceno BP, Carelli EF, Honorato DC, Facure JJ: The predictive value of cerebrospinal fluid tap-test in normal pressure hydrocephalus. Arq Neuropsiquiatr 55:179-185, 1997

13. de Laat KF, van Norden AG, Gons RA, van Oudheusden LJ, van Uden IW, Bloem BR, et al: Gait in elderly with cerebral small vessel disease. Stroke 41:1652-1658, 2010

14. Graff-Radford NR, Godersky JC: Normal-pressure hydrocephalus. Onset of gait abnormality before dementia predicts good surgical outcome. Arch Neurol 43:940-942, 1986

15. Ishikawa M, Hashimoto M, Kuwana N, Mori E, Miyake H, Wachi A, et al: Guidelines for management of idiopathic normal pressure hydrocephalus. Neurol Med Chir (Tokyo) 48 (Suppl):S1-S23, 2008

16. Jaraj D, Rabiei K, Marlow T, Jensen C, Skoog I, Wikkels $\varnothing$ $\mathrm{C}$ : Prevalence of idiopathic normal-pressure hydrocephalus. Neurology 82:1449-1454, 2014

17. Kerr B, Condon SM, McDonald LA: Cognitive spatial processing and the regulation of posture. J Exp Psychol Hum Percept Perform 11:617-622, 1985

18. Klinge P, Hellström P, Tans J, Wikkels $\varnothing$ C: One-year outcome in the European multicentre study on iNPH. Acta Neurol Scand 126: $145-153,2012$

19. Kressig RW, Herrmann FR, Grandjean R, Michel JP, Beauchet O: Gait variability while dual-tasking: fall predictor in older inpatients? Aging Clin Exp Res 20:123-130, 2008

20. Kristensen B, Malm J, Fagerland M, Hietala SO, Johansson B, Ekstedt J, et al: Regional cerebral blood flow, white matter abnormalities, and cerebrospinal fluid hydrodynamics in patients with idiopathic adult hydrocephalus syndrome. J Neurol Neurosurg Psychiatry 60:282-288, 1996

21. Lundin F, Tisell A, Leijon G, Leinhard OD, Davidsson L, Grönqvist A, et al: Preoperative and postoperative 1H-MR spectroscopy changes in frontal deep white matter and the thalamus in idiopathic normal pressure hydrocephalus. J Neurol Neurosurg Psychiatry 84:188-193, 2013

22. Malm J, Kristensen B, Fagerlund M, Koskinen LO, Ekstedt $\mathrm{J}$ : Cerebrospinal fluid shunt dynamics in patients with idiopathic adult hydrocephalus syndrome. J Neurol Neurosurg Psychiatry 58:715-723, 1995
23. Marmarou A, Bergsneider M, Klinge P, Relkin N, Black PM: The value of supplemental prognostic tests for the preoperative assessment of idiopathic normal-pressure hydrocephalus. Neurosurgery 57 (3 Suppl):S17-S28, ii-v, 2005

24. Marmarou A, Takagi H, Shulman K: Biomechanics of brain edema and effects on local cerebral blood flow. Adv Neurol 28:345-358, 1980

25. Mathew NT, Hartmann A, Meyer JS: The use of regional cerebral blood flow measured with the gamma camera in neurological diagnosis. Int J Neurol 11:194-205, 1977

26. Meyer JS, Tachibana H, Hardenberg JP, Dowell RE Jr, Kitagawa Y, Mortel KF: Normal pressure hydrocephalus. Influences on cerebral hemodynamic and cerebrospinal fluid pressure-chemical autoregulation. Surg Neurol 21:195203, 1984

27. Middleton A, Fritz SL, Lusardi M: Walking speed: the functional vital sign. J Aging Phys Act 23:314-322, 2015

28. Middleton A, Fulk GD, Beets MW, Herter TM, Fritz SL: Self-selected walking speed is predictive of daily ambulatory activity in older adults. J Aging Phys Act [epub ahead of print], 2015

29. Momjian S, Owler BK, Czosnyka Z, Czosnyka M, Pena A, Pickard JD: Pattern of white matter regional cerebral blood flow and autoregulation in normal pressure hydrocephalus. Brain 127:965-972, 2004

30. Nasreddine ZS, Phillips NA, Bédirian V, Charbonneau S, Whitehead V, Collin I, et al: The Montreal Cognitive Assessment, MoCA: a brief screening tool for mild cognitive impairment. J Am Geriatr Soc 53:695-699, 2005

31. Owler BK, Pena A, Momjian S, Czosnyka Z, Czosnyka M, Harris NG, et al: Changes in cerebral blood flow during cerebrospinal fluid pressure manipulation in patients with normal pressure hydrocephalus: a methodological study. J Cereb Blood Flow Metab 24:579-587, 2004

32. Pashler H: Dual-task interference in simple tasks: data and theory. Psychol Bull 116:220-244, 1994

33. Ravdin LD, Katzen HL, Jackson AE, Tsakanikas D, Assuras S, Relkin NR: Features of gait most responsive to tap test in normal pressure hydrocephalus. Clin Neurol Neurosurg 110:455-461, 2008

34. Relkin N, Marmarou A, Klinge P, Bergsneider M, Black PM: Diagnosing idiopathic normal-pressure hydrocephalus. Neurosurgery 57 (3 Suppl):S4-S16, ii-v, 2005

35. Rogers A, Bichsel D, Momjian S: Deformation of the corona radiata and internal capsule in normal pressure hydrocephalus. Neurochirurgie 60:216-221, 2014

36. Srikanth V, Phan TG, Chen J, Beare R, Stapleton JM, Reutens DC: The location of white matter lesions and gait-a voxel-based study. Ann Neurol 67:265-269, 2010

37. Stolze H, Drücke H, Kuhtz-Buschbeck J Jöhnk K, Illert M, Deuschl G: Gait analysis in normal pressure hydrocephalus-which parameters respond to the CSF-tap test? Electroencephalogr Clin Neurophysiol 106 (Suppl 1):61, 1998 (Abstract PS7-1)

38. Stolze H, Kuhtz-Buschbeck JP, Drücke H, Jöhnk K, Diercks $\mathrm{C}$, Palmié $\mathrm{S}$, et al: Gait analysis in idiopathic normal pressure hydrocephalus - which parameters respond to the CSF tap test? Clin Neurophysiol 111:1678-1686, 2000

39. Stolze H, Kuhtz-Buschbeck JP, Drücke H, Jöhnk K, Illert M, Deuschl G: Comparative analysis of the gait disorder of normal pressure hydrocephalus and Parkinson's disease. J Neurol Neurosurg Psychiatry 70:289-297, 2001

40. Sudarsky L, Simon S: Gait disorder in late-life hydrocephalus. Arch Neurol 44:263-267, 1987

41. Tamaki N, Shirakuni T, Ehara K, Matsumoto S: Characterization of periventricular edema in normal-pressure hydrocephalus by measurement of water proton relaxation times. $\mathbf{J}$ Neurosurg 73:864-870, 1990

42. Thomas G, McGirt MJ, Woodworth G, Heidler J, Rigam- 
onti D, Hillis AE, et al: Baseline neuropsychological profile and cognitive response to cerebrospinal fluid shunting for idiopathic normal pressure hydrocephalus. Dement Geriatr Cogn Disord 20:163-168, 2005

43. Tombaugh TN, McIntyre NJ: The mini-mental state examination: a comprehensive review. J Am Geriatr Soc 40:922935, 1992

44. Virhammar J, Cesarini KG, Laurell K: The CSF tap test in normal pressure hydrocephalus: evaluation time, reliability and the influence of pain. Eur J Neurol 19:271-276, 2012

45. Virhammar J, Laurell K, Ahlgren A, Cesarini KG, Larsson EM: Idiopathic normal pressure hydrocephalus: cerebral perfusion measured with pCASL before and repeatedly after CSF removal. J Cereb Blood Flow Metab 34:1771-1778, 2014

46. Wikkels $\varnothing$ C, Andersson H, Blomstrand C, Lindqvist G: The clinical effect of lumbar puncture in normal pressure hydrocephalus. J Neurol Neurosurg Psychiatry 45:64-69, 1982

47. Wikkelsö C, Andersson H, Blomstrand C, Lindqvist G, Svendsen P: Normal pressure hydrocephalus. Predictive value of the cerebrospinal fluid tap-test. Acta Neurol Scand 73:566-573, 1986

48. Woollacott M, Shumway-Cook A: Attention and the control of posture and gait: a review of an emerging area of research. Gait Posture 16:1-14, 2002

\section{Disclosures}

The authors report no conflict of interest concerning the materi- als or methods used in this study or the findings specified in this paper.

\section{Author Contributions}

Conception and design: Schniepp, Trabold, Wuehr, Peraud, Brandt, Dieterich, Jahn. Acquisition of data: Schniepp, Trabold, Romagna, Akrami, Hesselbarth. Analysis and interpretation of data: all authors. Drafting the article: Schniepp, Trabold, Akrami, Hesselbarth, Wuehr, Peraud, Brandt, Dieterich, Jahn. Critically revising the article: Schniepp, Trabold, Akrami, Wuehr, Peraud, Brandt, Dieterich, Jahn. Reviewed submitted version of manuscript: Schniepp, Trabold. Approved the final version of the manuscript on behalf of all authors: Schniepp. Statistical analysis: Schniepp, Akrami, Hesselbarth, Wuehr, Jahn. Administrative/ technical/material support: Schniepp, Romagna, Hesselbarth, Wuehr, Brandt, Dieterich, Jahn. Study supervision: Schniepp, Trabold, Romagna, Wuehr, Peraud, Brandt, Dieterich, Jahn.

\section{Supplemental Information \\ Online-Only Content}

Supplemental material is available with the online version of the article.

Supplemental Tables. http://thejns.org/doi/suppl/10.3171/ 2015.12.JNS151663.

\section{Correspondence}

Roman Schniepp, Department of Neurology, University of Munich, Marchioninistrasse 15, Munich 81377, Germany. email: roman.schniepp@med.uni-muenchen.de. 